\title{
Impact of Neuro Linguistic Programming on Social Communities through Visual Kinesthetic Dissociation
}

\author{
Arun Kumar \\ AP, Research Scholar \\ FET, CSE, MRIIRS
}

\author{
Supriya P. Panda, PhD \\ Professor, HOD \\ FET, CSE, MRIIRS
}

\begin{abstract}
Neuro-Linguistic Programming (NLP) techniques useful for the growth and development of social communities. Visual kinesthetic dissociation (V/KD) technique used to affect the behavior patterns of the millennials. V/KD process used by the NLP trainers to know the symptoms of phobia, fear and stress disorders can impact many social communities. The study based on the V/KD explains the pattern of behavior and current states of the fearful mind. Visual-kinesthetic dissociation also known as the Rewind technique finds a way to overcome the states among people suffering from fear and phobia. The research aims to provide awareness in social communities to change the patterns of the mind. This paper explains the working of the V/KD process and at the same time analyzes patterns and finds the solutions.
\end{abstract}

\section{Keywords}

Neuro Linguistic Programming (NLP), NLP Techniques, Visual Kinesthetic disassociation (V/KD), Millennials, Reframing, Bayes Theorem, Python

\section{INTRODUCTION}

Neuro-linguistic programming is a modeling process that was founded in the 1970s by Richard Bandler and Dr. John Grinder. This process allows physiotherapists and hypnotherapists to change the programming of the human mind. This method is useful in the treatment of many patients affected by fear and phobia. Today in the world of Information technology, millennials go faster to gain knowledge through every field of the market. In every country and every society, the millennials and the social communities are the key factors of the progress [5]. The study of NLP makes the tremendous impact on modern psychology of many professional and personal roles of the society. Neurolinguistic programming recommended for many areas of the social communities for growth and development purposes. For this neuro -linguistic programming help millennials to improve the awareness around their environment. Neurolinguistic programming teaches millennials to listen to their minds and the messages that it conveys. Neuro-linguistic programming is a better approach to understand people about their feelings beliefs and behavior. It can also teach the way to improve effective communication among them. Learning Neuro-linguistic programming can be a life-changing experience for their growth and development [2]. Many of the areas like administrators, managers, executives, trainers and counselors can learn neuro-linguistic programming to improve progress in their work environment. This can also improve their personal and professional relations through the technique of neuro-linguistic programming. Neuro-linguistic programming teaches them how to balance their feelings in their professional and personal environment $[3,4]$.

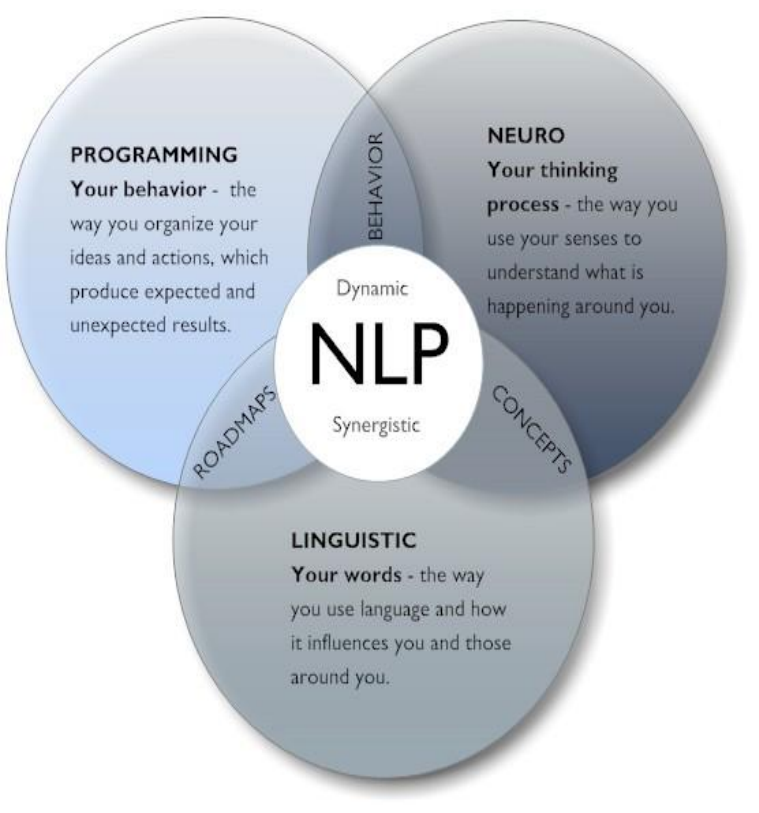

Figure 1 : Neuro- Linguistic Programming: Internet Source

There are many techniques in NLP like anchoring reframing, visual Kinetic dissociation and many more, which can help millennials and other communities to improve their way of thinking and training their mind according to the current scenarios [6]. We all are living in the changing world in which in every moment we have to face new challenges. Every time the minds need to change according to the challenges faced created through many situations and conditions. This paper focuses on the most prominent technique of neuro-linguistic programming useful for the growth and development of millennials and social communities.

\section{NLP SUBMODALITIES}

Submodalities are an important term used in the world of NLP. Submodalities concerned with the five senses of the real world that we see, touch, smell, speak and hear. Submodalities help people to sense the world around them. It is an important aspect used in NLP techniques to change the patterns of human behavior. Submodalities are the key source to work upon the human senses and change the patterns according to the real-world scenarios. NLP defines the quality of each modality which can be useful to overcome the fear and phobia among millennials and other communities [7]. NLP use submodalities to recall the images of past memories and create new images to change the belief system of social communities. 
Table.1: NLP Submodalities: Internet Source

\begin{tabular}{|l|l|l|}
\hline \multicolumn{1}{|c|}{ Visual } & \multicolumn{1}{|c|}{ Auditory } & \multicolumn{1}{c|}{ Kinesthetic } \\
\hline Black \& White or Colour & Loud or Soft & Strong or Weak \\
Near or Far & Near or Far & Large Area or Small Area \\
Location & Weight: Heavy or Light \\
Bright or Dim & Location & Location \\
Size of Picture & Stereo or Mono & Texture: Smooth or \\
Associated / Dissociated & Fast or Slow & Rough \\
Focused or Defocused & High or Low Pitch & Constant or Intermittent \\
Framed or Unbounded & Verbal or Tonal & Temperature: Hot or \\
Movie or Still & Rhythm & Cold \\
If a Movie- & Pauses & Shape \\
Fast/Normal/Slow & Clarity & Size \\
3 Dimensional or Flat & & Pressure \\
& & Vibration \\
\hline
\end{tabular}

NLP defines the three types of submodalities as shown in Table1. The first modality relates to the visual effect of the real-world picture. This modality shows the property of a visual image, its colour, distance, location, size association, the focus, and its dimension. The second modality shows the audio effect of the real-world situation [8]. This shows the property of audio, its sound, where it comes from ;it is internal or external, fast or slow, high or low, rhythm and its clarity. The last one is the Kinetic property which relates to the physical posture of the image, strong or weak, the weight of the picture, its texture and also shows the temperature conditions where it resides.

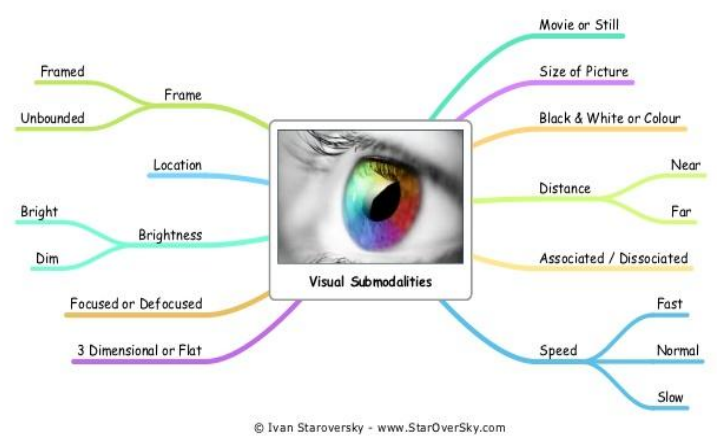

Figure.2: Visual Submodalities: Internet Source

Figure. 2 shows the concept of visual submodalities. This figure described the properties of a visual image. NLP is useful for the millennials to recall the visuals according to the above properties of the image [9]. The visual property of any image can affect the patterns of mind. It can also impact on the belief system of millennials and social communities.

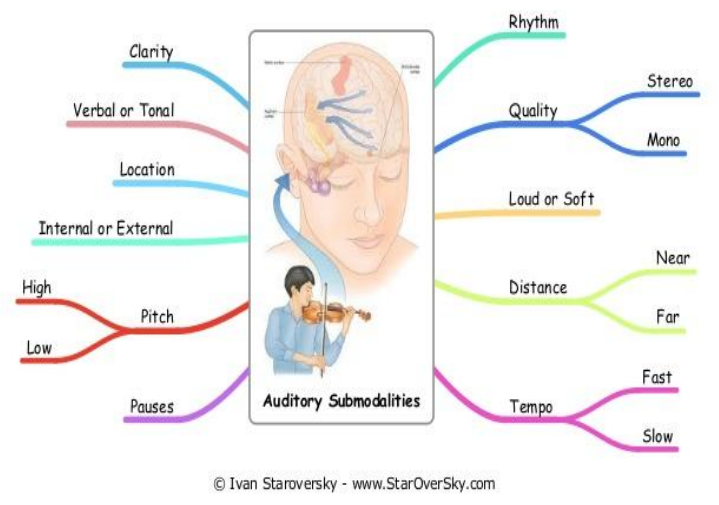

Figure.3: Auditory Submodalities: Internet Source
Figure. 3 shows the concept of auditory submodalities. This figure described the properties of an audible sound. NLP helps people to recognize the audible sounds stored in the mind according to the above properties of the audio. The audible property of any sound can effect to patterns of the behavior of mind.

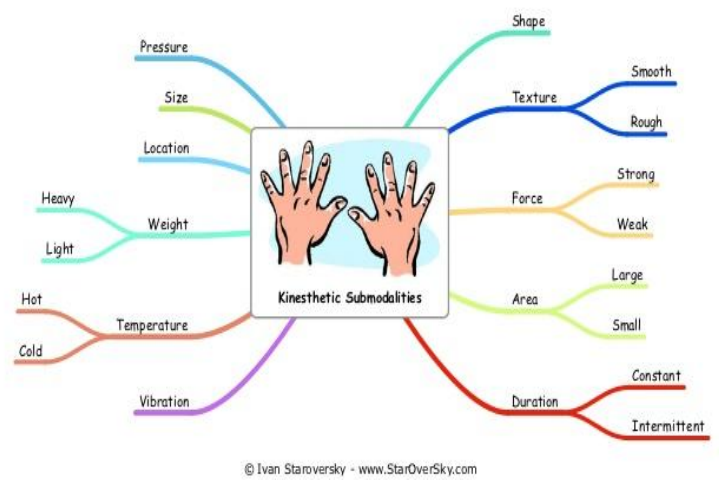

Figure.4: Kinesthetic Submodalities: Internet Source

Fig.4 shows the concept of kinesthetic sub modalities. This figure described the properties of a physical texture or a posture. NLP helps people to recognize the postures stored in the mind according to the above properties of the kinesthetic image. The Kinesthetic property of any posture can effect on the patterns of behavior of human mind. It can also impact on belief system of millennials and other social communities.

\section{REFRAMING WITH NLP}

In the real world, people always come across the interaction process. The interaction process can create conflicts with the outside environment. Conflict can arise for many reasons through disagreements, external and internal pressure, personality disputes and disbeliefs. Neuro-Linguistic programming provides a way to reduce the conflict and establish effective communication between the social communities. Millennials are constantly struggling with effective communication with their parents, teachers and elder ones. Neuro-linguistic programming provides skills to manage the conflict among millennials and also helps to prevent situations from getting worse. Through NLP, the human resources team trained to learn how to handle conflict with customers and third parties [13]. Neuro-linguistic programming used to understand the differences among various social communities to improve effective communication between them. Neuro-linguistic programming can reframe the belief system and behavior patterns among millennials. Reframing is the process which can affect the old the mind and frames the new patterns as required by the current scenarios.

Table.2: V-K Dissociation Procedure

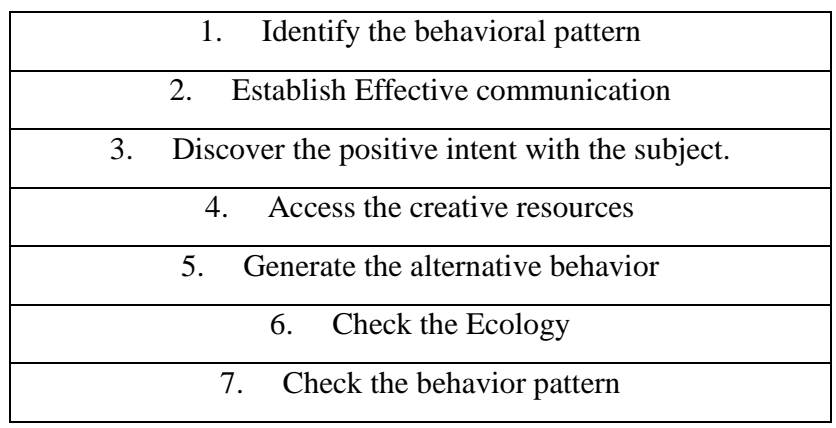




\section{VISUAL KINESTHETIC DISSOCIATION}

The human mind collects information from various sources. These sources based on the association and dissociation of the information. The information connected to the feelings and beliefs relates to associated information. The dissociated information related to the logical pattern of the mind. The processing of information always connected to the belief system of the human mind. Association allows people to experience life at the first point of contact. People associated with any information through feelings and emotions. On the other hand, dissociation is a careful approach. Dissociation provides a way to decide how to respond or react to a situation. In dissociation, the aim is to learn from the situation and do not allow to induce emotional responses. Too much associating with the events leads to the risk of becoming an emotional cripple [8]. Too much dissociating from the events leads to the emotionally incompetent. It will block the ability to relate the events and content at a personal or emotional level. People become habitual to many traumatic and phobic events. Visual kinesthetic dissociation is a powerful technique that can help individuals to recover from past experiences and theses phobic events. Another name of visual kinesthetic dissociation is the rewind technique. It can be used for the treatment of stress disorders, traumatic events, panic attacks, and phobias [9]. V-K Dissociation helps to reduce the effects of past memories and traumatic events. People can suffer from the phobia of animals, fear of people, objects treated by this technique. V-K Dissociation reprograms the mind of the participants and gets back it to the original state. Participants affirm their back state of the mind and instruct well to be able to end the effect of phobic events [15].

\section{V-K DISSOCIATION PROCEDURE}

The V/KD Process is very effective involves the participants to think about the traumatic impact and feelings. This process allows the neurolinguistics experts to study and measure the feelings and emotions of the traumatic events. This process gives the clarity of the current state of mind. This process can recognize the edge of the therapy and can step back when needed. V/KD determines the state of the participants and at the same time, they can get back to any stable state. Table 2 describes the steps of the V/KD procedure than can be used in the NLP workshops to find the appropriate behavior pattern of the participants of the workshop. NLP trainers follow the same instruction as described in the $\mathrm{V} / \mathrm{KD}$ process.

\subsection{Visualization}

Ask the participants to imagine them watching a movie. Instead, to see the characters they see a picture of themselves as a character of the same mental image but do not involve any emotional sentiment. This is ideal with the visualization of memories.

\subsection{Dissociation}

Ask the participants to visualize the task as a dissociated observer. Also, see the colors of the image. Watch everything in black and white, imagine turning everything into colors. Color emphasizes the senses, what we are feeling, hearing and seeing.

\subsection{Reframing}

Ask the participants to visualize the same picture but now in reverse order. The idea here is to reframe horrific sentiments and emotions to neutralize them. The next attempt of this process is systematic desensitization.

\subsection{Anchoring}

Ask the participants to follow the instructions given by the NLP trainer. These instructions based on the V/KD process. As shown in Table 2 all the participants follow the steps in the same manner. Step by step they follow the process and doing the same as instructed. This process based on the behavior patterns of the mind. The behavior patterns recognized by the trainers and work on it as required according to the participants' needs. V/KD technique is useful to overcome fears, phobias, and stress disorders. The above steps in V/KD identify the behavior pattern of the participants. Then trainers establish effective communication with them when every time trainers find the positive intent in the situations described by the participants. After that trainers find the appropriate method to change the pattern of the mind. These methods relate to NLP techniques. V/KD generates alternate behavior among all the participants. This behavior is somehow temporary but tested and applying for many situations. Trainers maintain the ecology of the whole process to create the appropriate environment and check the behavior pattern.

\section{NLP WORKSHOP EXPERIMENTAL RESULTS}

Neuro-linguistic programming workshops experimental results are shown in Table 3, Table 4 and Table 5.

Table.3: Results of V/KD feeling technique

\begin{tabular}{|c|c|c|c|}
\hline & $\begin{array}{c}\text { Upfront } \\
\text { appreciatio } \\
\mathbf{n}\end{array}$ & $\begin{array}{c}\text { Negative } \\
\text { Sentimen } \\
\mathbf{t} \\
\text { V/KD feeling } \\
\text { correctio } \\
\text { technique }\end{array}$ & $\begin{array}{c}\text { by NLP } \\
\text { coach/trai } \\
\text { ner }\end{array}$ \\
\hline 1 & $80 \%$ & $99 \%$ & $20 \%$ \\
\hline 2 & $50 \%$ & $70 \%$ & $10 \%$ \\
\hline 3 & $80 \%$ & $90 \%$ & $30 \%$ \\
\hline 4 & $80 \%$ & $80 \%$ & $30 \%$ \\
\hline 5 & $60 \%$ & $80 \%$ & $20 \%$ \\
\hline
\end{tabular}

Table.4: Results of V/KD Imagination technique

\begin{tabular}{|c|c|c|c|}
\hline S.No. & $\begin{array}{c}\text { Upfront } \\
\text { appreciatio } \\
\mathbf{n}\end{array}$ & $\begin{array}{c}\text { Result V/KD } \\
\text { Imagination } \\
\text { technique }\end{array}$ & $\begin{array}{c}\text { Negative } \\
\text { Sentimen } \\
\mathbf{t} \\
\text { correctio } \\
\text { n by NLP } \\
\text { coach/trai } \\
\text { ner }\end{array}$ \\
\hline 1 & $80 \%$ & $80 \%$ & $30 \%$ \\
\hline 2 & $50 \%$ & $80 \%$ & $10 \%$ \\
\hline 3 & $80 \%$ & $80 \%$ & $40 \%$ \\
\hline 4 & $80 \%$ & $60 \%$ & $32 \%$ \\
\hline 5 & $60 \%$ & $70 \%$ & $25 \%$ \\
\hline
\end{tabular}


Table.5: Results of V/KD thinking technique.

\begin{tabular}{|c|c|c|c|}
\hline & Upfront & $\begin{array}{c}\text { Result } \\
\text { V/KD } \\
\text { thinking } \\
\text { technique } \\
\text { as } \\
\text { reported } \\
\text { by subject }\end{array}$ & $\begin{array}{c}\text { Negative } \\
\text { Sentimen } \\
\text { t } \\
\text { correctio } \\
\text { n by NLP } \\
\text { coach/trai } \\
\text { ner }\end{array}$ \\
\hline 1 & $80 \%$ & $60 \%$ & $30 \%$ \\
\hline 2 & $50 \%$ & $80 \%$ & $20 \%$ \\
\hline 3 & $80 \%$ & $70 \%$ & $40 \%$ \\
\hline 4 & $80 \%$ & $85 \%$ & $27 \%$ \\
\hline 5 & $60 \%$ & $70 \%$ & $35 \%$ \\
\hline
\end{tabular}
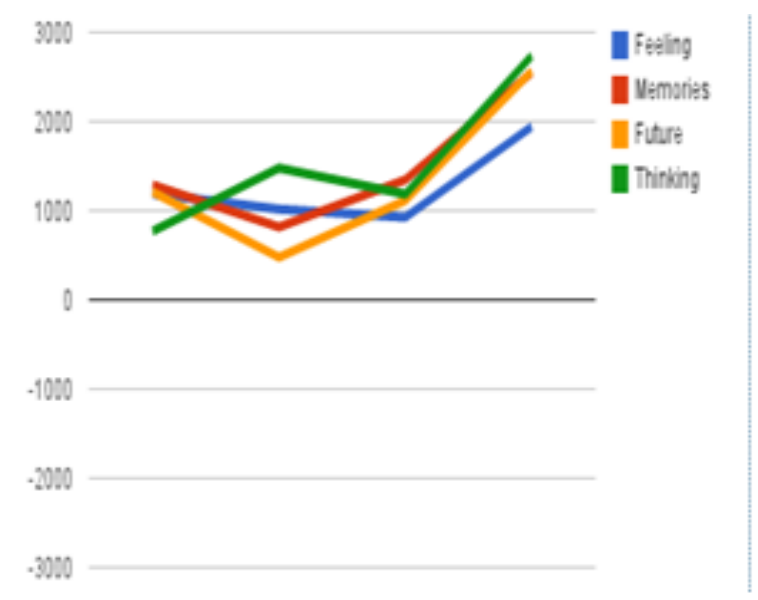

NLP Trainer \#1: www.purenlp.com
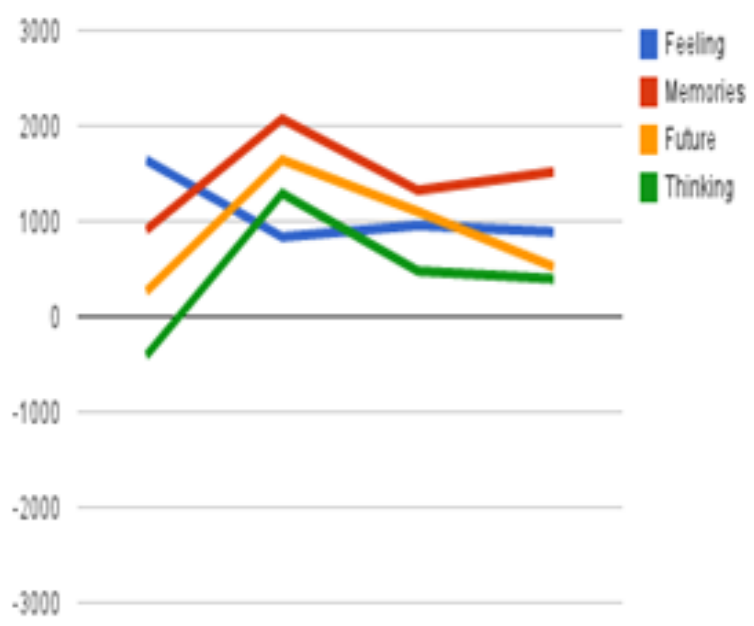

\section{NLP Trainer \# 2: www.purenlp.com}

The above graphical representation shows the results of the feeling, imagination and thinking techniques of NLP. The mathematical results are shown in the tabular form. Table. 3 shows the results of the V/KD feeling technique. Table.4 shows the results of the V/KD imagination technique of NLP. Table.5 shows the V/KD thinking results of NLP. The parameters of negative sentiment correction percentage, the upfront appreciation are shown in the tables. The results contain the sample data of five participants. The workshop is managed by NLP trainers. The main source of the workshop is the visual kinesthetic dissociation technique. This technique is applied to the 81 participants out of whom 77 participants get excellent results. The success rate is 0.95 calculated by using Bayesian formula. The above graphs show the results in a graphical pattern by using Jupyter notebook in python. These above tabular and graphical results are based on the behavior patterns of the practitioners. V/KD process used all the combinations to affects the patterns of the mind. NLP trainers recognize the patterns and check the before and aftereffects of the mind and then apply V/KD to change it as per the situations and requirements of the participants. This shows that $\mathrm{V} / \mathrm{KD}$ is the most prominent technique and can be useful for many social communities for the growth and development point of view.

\section{CONCLUSION AND FUTURE SCOPE}

Visual kinesthetic dissociation creates a tremendous effect on the behavior pattern of the mind. In this paper, the V/KD process is applied to the participants suffering from fear, phobia and many stress disorders to make a change in the patterns of behavior. NLP workshops are the main source to overcome such traumatic situations. The graphical representation and tabular methods are used to represent participants' satisfactory results. The results are based on the discussions with NLP workshop practiniores. Participants express feelings and sentiments based on the V/KD process. The next phase of the research work will be based on the Meta program analysis of neuro linguistic programming.

\section{REFERENCES}

[1] Ahmad, K. Z. (2017, December), Improving Emotional Intelligence (EI) using Neuro Linguistic Programming (NLP) techniques, In International Conference on Advances in Business, Management and Law (ICABML) ,Vol. 1, No. 1, pp. 172-183.

[2] Carey, J., Churches, R., Hutchinson, G., Jones, J., \& Tosey, P. (2010). Neuro-Linguistic Programming and Learning: Teacher Case Studies on the Impact of NLP in Education, Online Submission.

[3] Florina, G.E., Teodor, G.V., Cornelia, P.S. and Marin, D., 2015, Neuro-linguistic programming and the relationship between attention and anxiety in Alpine skiing juniors, Procedia-Social and Behavioral Sciences, 191, pp.1634-1638.

[4] Gaspar, R., Pedro, C., Panagiotopoulos, P. and Seibt, B., 2016. Beyond positive or negative: Qualitative sentiment analysis of social media reactions to unexpected stressful events, Computers in Human Behavior, 56, pp.179-191.

[5] Gibson, B. (2011). The Complete Guide to Understanding and Using NLP: Neuro-linguistic Programming Explained Simply. Atlantic Publishing Company.

[6] Grosu, E. F., Grosu, V. T., Preja, C. A., \& Iuliana, B. B. ,2014, Neuro-linguistic Programming based on the Concept of Modelling, Procedia-Social and Behavioral Sciences, 116, 3693-3699.

[7] Grinder, J., \& Pucelik, F. (2012), the Origins of Neuro Linguistic Programming, Crown House Publishing. 
[8] Hosseinzadeh, E. and Baradaran, A., 2015, Investigating the Relationship between Iranian EFL Teachers' Autonomy and Their Neuro-Linguistic Programming, English Language Teaching, 8(7), pp.68-75.

[9] Karunaratne, M., 2010, Neuro-linguistic programming and application in treatment of phobias, Complementary therapies in clinical practice, 16(4), pp.203-207.

[10] Kök, İ., 2014, Listening comprehension achievement and brain dominance, Procedia-Social and Behavioral Sciences, 122, pp.329- 334.

[11] Kotera, Y., \& Sweet, M. (2019), Comparative evaluation of neurolinguistic programming. British Journal of Guidance \& Counselling, 1-13.

[12] Lashkarian, A. and Sayadian, S. ,2015, The effect of NeuroLinguistic Programming techniques on young Iranian EFL Learners' motivation, learning improvement, and on teacher's success, ProcediaSocial and Behavioral Sciences, 199, pp.510-516.

[13] Louis Sinclair. (2019), A Complete Guide to Neuro Linguistic Programming.

[14] Neidhardt, J., Rümmele, N. and Werthner, H., 2017, Predicting happiness: user interactions and sentiment analysis in an online travel forum, Information Technology \& Tourism, 17(1), pp.101-119.

[15] Learners' motivation, learning improvement, and on teacher's success. Procedia-Social and Behavioral Sciences, 199, pp.510-516.

[16] Neidhardt, J., Rümmele, N. and Werthner, H., 2017, predicting happiness: user interactions and sentimen analysis in an online travel forum, Information Technology \& Tourism, 17(1), pp.101-119.

[17] Passmore, J. and Rowson, T., 2019, Neuro-linguistic programming: A review of NLP research and the application of NLP in coaching. International Coaching Psychology Review, 14(1), p.57.

[18] Roderique-Davies, G., 2009, Neuro-linguistic programming: cargo cult psychology, Journal of applied research in higher education, 1(2), pp.58-63.

[19] Vanmassenhove, E., Cabral, J.P. and Haider, F., 2016, Prediction of emotions from text using sentiment analysis for expressive speech synthesis, In 9th ISCA Speech Synthesis Workshop 13-15 Sep 2016, Sunnyvale, USA , pp.21-26. 